\title{
Immunological Implications of Physical Inactivity among Older Adults during the COVID-19 Pandemic
}

\author{
Anthony Damiot ${ }^{\mathrm{a}}$ Ana Jéssica Pinto ${ }^{\mathrm{a}}$ James E. Turner ${ }^{\mathrm{b}}$ Bruno Gualano ${ }^{\mathrm{a}, \mathrm{c}}$ \\ ${ }^{a}$ Applied Physiology and Nutrition Research Group, School of Physical Education and Sport, Rheumatology Division, \\ Faculdade de Medicina FMUSP, Universidade de São Paulo, São Paulo, Brazil; ${ }^{b}$ Department for Health, University of \\ Bath, Bath, UK; ${ }^{C}$ Food Research Center, University of São Paulo, São Paulo, Brazil
}

\section{Keywords}

Older adults $\cdot$ Health $\cdot$ Immunology $\cdot$ Inflammation .

Coronavirus

\section{Abstract}

Social distancing has been adopted worldwide to control severe acute respiratory syndrome coronavirus 2 (SARS-CoV-2) transmission. Social isolation is likely to lead to a decline in physical activity, which could result in immune system dysfunction, thereby increasing infection susceptibility and exacerbating the pathophysiology of conditions that are common among older adults, including cardiovascular disease, cancer, and inflammatory disorders. Older adults and people living with these comorbidities are at a greater risk for complications during coronavirus disease 2019 (COVID-19). In this review, we discuss the negative impact of physical inactivity on immune function and showcase evidence that regular physical activity may be an effective strategy to counter some of the deleterious effects of social isolation. Furthermore, we briefly highlight key research questions in exercise immunology, with a focus on older adults in the context of COVID-19. Although it is worth emphasizing that there is no direct evidence that physical activity can prevent or treat COVID-19, promoting an active lifestyle is a key intervention to counteract the effects of social isolation, especially in older adults and other at-risk individuals, such as those living with chronic diseases associated with ageing and lifestyle.

(c) 2020 S. Karger AG, Basel

\section{Introduction}

The emergence of severe acute respiratory syndrome coronavirus 2 (SARS-CoV-2) and the complications caused by coronavirus disease 2019 (COVID-19) are a global public health challenge. The pathophysiology of SARS-CoV-2 infection closely resembles cases from the 2002-2003 epidemic of SARS-CoV, with aggressive inflammatory responses resulting in damage to the lungs [1]. Disease severity is not only due to viral infection but it is strongly influenced by the characteristics of the host immune response. Indeed, SARS-CoV-2 infection and the destruction of lung cells trigger a local immune response. Innate immune cells including local macrophages, and monocytes recruited from blood, respond to karger@karger.com

www.karger.com/ger

(C) 2020 S. Karger AG, Base

Karger'
Prof. Dr. Bruno Gualano

Applied Physiology and Nutrition Research Group, Rheumatology Division Faculdade de Medicina FMUSP, Universidade de São Paulo

Ave. Dr. Arnaldo, 455, $3^{\circ}$ andar, Sao Paulo, SP 01246-903 (Brazil) gualano@usp.br 


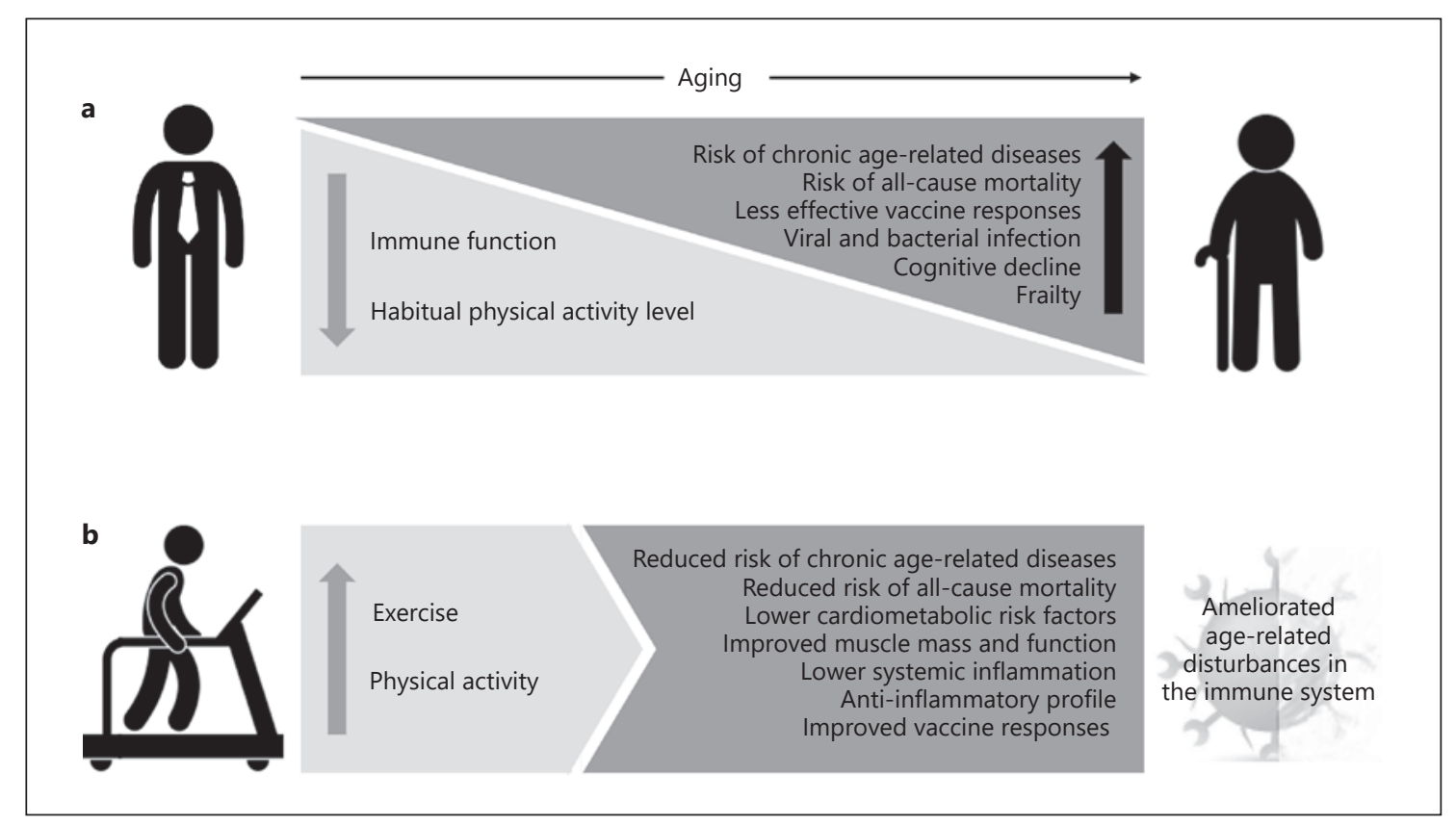

Fig. 1. Impact of aging (a) and physical activity (b) on immune function and morbimortality.

the infection releasing cytokines, priming an adaptive immune response from T cells and B cells [1]. In most cases, this immune response is capable of resolving the infection. However, in some cases, an exaggerated production of cytokines, also known as a "cytokine storm," can lead to sepsis, which is responsible for $28 \%$ of the total fatal cases of COVID-19. In such cases, exacerbated inflammation leads to multi-organ damage, affecting mostly pulmonary, cardiac, hepatic, vascular, and renal systems [1].

In the absence of evidence-based treatments or vaccines to counter SARS-CoV-2, social distancing has been adopted worldwide to control virus transmission; however, isolation measures are not without adverse effects. Periods of confinement can lead to physical dysfunction and mental distress, partially attributed to reductions in habitual physical activity [2]. Physical inactivity accumulated over weeks and months can lead to immune system dysfunction, which could, in principle, increase infection susceptibility and exacerbate the pathophysiology of conditions that are common among older adults, including cardiovascular disease, cancer and inflammatory disorders [3]. Older adults and people living with these comorbidities are at a greater risk for complications during COVID-19 disease. Given that ageing is also associated with a decline in physical activity and alterations in immune function $[4,5]$, it is plausible that there is an interaction between these age-associated changes and COVID-19 disease pathogenesis. In this brief review, we discuss the negative impact of physical inactivity on immune function and showcase evidence that regular physical activity may be an effective strategy to counter some of the deleterious effects of social isolation. Furthermore, we highlight key research questions in exercise immunology, with a focus on older adults in the context of COVID-19.

\section{Physical Inactivity and the Immune System}

The majority of research conducted over the past century has investigated how being physically active affects the immune system [6] (Fig. 1b). However, evidence is beginning to accumulate that physical inactivity, and its consequences, such as adipose tissue accumulation and muscle dysfunction, deleteriously affect both innate and adaptive immunity. For example, physical inactivity accumulated over weeks, months, and years is associated with increased systemic inflammation (e.g., increased TNF- $\alpha$, IFN- $\gamma$, and CRP), impaired natural killer cell cytolytic activity [7], and reduced T-cell proliferation and cytokine production $[8,9]$, all of which can result in a loss of viral control $[10,11]$. It is important to emphasize that some understanding of how physical inactivity affects immune function comes from experiments conducted in space, so the effects of microgravity, radiation exposure, 
and psychological stress need to be considered. However, other studies have been conducted using space-flight analogs on Earth, consisting of head-down tilt bed rest in humans, or in animal models, using hind-limb unloading $[9,10,12]$. For example, in rodents, hind-limb unloading resulted in decreased responses of bone marrow cells to colony-stimulating factors, resulting in an alteration of leukocyte differentiation and activation following antigenic stimulation [13]. Other studies have shown that hind-limb unloading results in failure to generate longterm memory responses among $T$ cells leading to a loss of resistance to bacterial pathogens [14]. Human bed rest studies have also reported increases in systemic inflammation, alterations in leukocyte subset distribution, impaired T-cell IFN- $\gamma$ production, and impaired natural killer cell functions [13]. These immune system disturbances are thought to contribute to the development of urinary tract infections and reactivation of latent herpesviruses in astronauts during missions [13] or mild reactivation of Epstein-Barr virus and varicella zoster virus [15] during prolonged periods of physical inactivity. Further research that could be interpreted as supporting the concept that physical inactivity leads to impaired immune function comes from other settings, such as studies recruiting overweight or obese participants who are often physically inactive too. For example, people who are obese exhibit poor antibody responses to vaccination [16-18] and impaired lymphocyte proliferation following mitogenic stimulation [19]. These immunological effects likely lead to a greater risk of viral and bacterial infections and longer stays in hospital due to more frequent and prolonged complications following surgery $[20,21]$.

\section{Physical Activity and the Immune System}

Research over the past century has determined that being regularly physically active and undertaking bouts of exercise has a profound effect on the immune system [6]. Although since the 1980s there has been a debate over whether very large volumes or high intensities of exercise can suppress the immune system [22,23], it is now generally agreed upon that most forms of moderate- to vigorous-intensity exercise is beneficial for immune function [24]. Indeed, it is now accepted that bouts of exercise enhance immune surveillance. This is because exercise causes substantial hemodynamic changes (e.g., increases in cardiac output, vasodilation, and blood flow), which impose mechanical forces on the endothelium [11]. This causes leukocytes to demarginate and enter the free-flow-

Physical Inactivity, Aging, and COVID-19 ing circulation concomitantly with the release of catecholamines and glucocorticoids, following the activation of the sympathetic nervous system and the hypothalamicpituitary-adrenal axis [11]. This orchestrated response induces a striking 2- to 4-fold increase in total leukocytes (leukocytosis) and a redistribution of so-called effector cells between the blood compartment and the lymphoid and peripheral tissues [11]. Indeed, exercise preferentially mobilizes leukocyte subtypes with tissue-migrating characteristics and the capacity for rapid effector function, such as natural killer cells, CD8+ T cells, and neutrophils [11]. The rapid redistribution of immune cells with every exercise bout most likely enhances immune surveillance, reducing the chance of pathogens gaining a foothold and causing overt disease in regular exercisers. Many of the immunoenhancing effects of exercise are direct, including "priming" of the cells mobilized by exercise, improving their tissue migration capacity, cytotoxic function, and the ability to recognize antigens [11]. Other effects of exercise include immune cell metabolic reprogramming, anti-inflammatory signaling [11], and effects on the lymphatic system [25]. For example, although lymph is thought to primarily circulate due to intrinsic lymphatic pumping, extrinsic compression caused by skeletal muscle contraction, respiratory activity, and bodily movement also contribute [25]. Thus, being physically active may facilitate the circulation of lymph within the lymphatic vessels, further enhancing the ability of the immune system to respond to antigens [25].

Although the immune system responds to single bouts of exercise in a transitory way, it is likely that these effects accumulate over time and produce the immunological adaptations that happen with long-term exercise training [11] (Fig. 1b). For example, regular exercise has been shown to reduce chronic low-grade inflammation and has been linked to increased $\mathrm{T}$-cell proliferation and cytokine production following antigenic stimulation, increased neutrophil phagocytic activity, and increased natural killer cell cytolytic activity [11]. These changes indicate that regular, moderate-intensity exercise might be capable of improving, or at least maintaining, immunity across the life span [26]. Indeed, exercise training is capable of improving immune responses to influenza and pneumococcal vaccines, hastening the recovery following experimental rhinovirus infection [11], and reducing the incidence and the number and severity of symptoms associated with acute respiratory infections (e.g., upper respiratory tract infection) [24]. Long-term regular exercise training can also reduce the expression of Toll-like-receptors (especially TLR-4) on the surface of monocytes, pro- 
moting switching of the proinflammatory M1-like macrophages to the anti-inflammatory M2-like, a response that mitigates the monocyte-downstream inflammatory signaling cascades, resulting in anti-inflammatory actions [27]. Other longer-term but indirect effects of exercise on the immune system are largely attributable to changes in body composition and metabolism [11]. Reducing fat mass reduces inflammatory macrophage accumulation in adipose tissue, chronic low-grade inflammation, and inflammatory signaling cascades [28]. Reducing cholesterol content of cell membranes that accompanies changes in body composition may improve T-cell receptor signaling for antigen presentation, while improvements in cardiovascular and endothelial function with exercise may facilitate immune cell recirculation between the blood, the lymphoid, and peripheral tissues [11]. Finally, evidence that regular physical activity over a lifetime might prevent, delay, or limit the age-associated deterioration of immune competency - referred to as immunosenescence - is now beginning to accumulate $[5,26]$.

It is unknown how bouts of physical activity and exercise might influence the immune response to SARSCoV-2 infection, which could be a moot point given that individuals self-isolate and typically rest when an infection is suspected. However, there remains a possibility that individuals may undertake physical activity or exercise when unknowingly infected, especially if initially asymptomatic. Following SARS-CoV-2 infection, active replication, and release of virus causes the host cells to undergo pyroptosis and release "danger signals" recognized by neighboring epithelial cells, endothelial cells, and alveolar macrophages, which produce proinflammatory cytokines and chemokines. Additional tissue-resident macrophages are recruited, and monocytes and $\mathrm{T}$ cells extravasate from blood and other tissues, contributing to the inflammatory response. Thus, it is conceivable that this immune surveillance response might be enhanced by bouts of physical activity or exercise, facilitating the detection and elimination of SARS-CoV-2 infected cells and potentially limiting disease severity. For example, animal experiments using labelled cells have shown that the lungs are a site of immune cell extravasation following exercise [29]. However, if SARS-CoV-2 infection is suspected, resting would be the most cautious approach, given that it is the excessive accumulation of immune cells in the lungs, and an overproduction of proinflammatory cytokines, which leads to complications in the most severe cases of COVID-19 [1].

\section{Immunosenescence as a Modifiable Risk Factor for COVID-19?}

Immunosenescence is the decline in immune function that takes place with biological aging (Fig. 1a), which is influenced by environmental factors, such as viral infection, in particular cytomegalovirus, but probably also lifestyle $[5,26,30]$. Immunosenescence is characterized by particularly marked changes in the adaptive immune system, especially among $T$ cells and B cells [31]. Within the $\mathrm{T}$-cell compartment, there is a diminished thymic output of naive T cells from adolescence and continued differentiation into memory $\mathrm{T}$ cells with infections [31]. Some studies show that, overall, $\mathrm{T}$ cells exhibit a reduced proliferative capacity and cytokine production in response to stimulation by mitogens [30]. Other cells are also affected, including a decreased production of proinflammatory cytokines in response to Toll-like receptor agonists in myeloid dendritic cells [31]. Within the B-cell compartment, naive B cells decline, memory B cells accumulate, and there is a diminished diversity of the B-cell repertoire [31]. These effects on the cellular composition and function of the immune system lead to a greater incidence and severity of viral and bacterial infections among older adults [32, 33] and less effective vaccine responses [34] when compared to younger individuals. Importantly, immunosenescence has been linked to a higher risk of chronic diseases, frailty, and all-cause mortality [35]. Importantly, individuals exhibiting signs of immunosenescence may have poor control over the initial inflammatory response to infection and may have a poor ability to generate an efficient and robust adaptive immune response by effector T cells and B cells $[31,35]$. This may partially explain the suboptimal responses to infection and vaccination exhibited by older adults compared to younger counterparts [34]. Thus, it could be argued that immunosenescence predisposes older adults to a greater risk of complications caused by COVID-19 [36].

Encouragingly, evidence is beginning to accumulate showing that people who have been regularly active for the majority of their lifetime exhibit less pronounced features of immunosenescence. For example, one study compared 125 adults (55-79 years) who had maintained a high level of physical activity for much of their adult lives to lessactive 75 age-matched controls [4]. The frequency of B cells, naive $\mathrm{T}$ cells, and recent thymic emigrants were higher in active compared to inactive older adults. Some of these effects were thought to be driven by higher serum levels of the thymoprotective cytokine IL-7 and lower IL-6 among active older adults [4]. In addition, there is evi- 
dence that regular exercise training can improve influenza vaccine responses among older individuals [11]. For example, antibody titers to the $\mathrm{H} 1 \mathrm{~N} 1$ and $\mathrm{H} 3 \mathrm{~N} 2$ strains of the influenza A virus were enhanced in older adults immunized with a trivalent influenza vaccine after a 10-month aerobic exercise training intervention [11]. Likewise, community-dwelling older adults engaged in a 10-month, cardiovascular exercise training program had increased seroprotection rates compared to nonexercising age-matched controls up to 24 weeks after receiving an influenza vaccine [11]. Studies showing improvements in vaccine responses following exercise training are considered to be robust evidence showcasing how the immune system can be positively affected by exercise [37]. Although it might be expected for very active people to exhibit slower immunological ageing compared to inactive people, interaction between physical activity or exercise with individual components or drivers of immunosenescence - such as cytomegalovirus - are more nuanced [38]. For example, individuals latently infected with cytomegalovirus exhibit exaggerated exercise-induced T-cell immune surveillance, which might improve the detection of infections and damaged cells but could also amplify inflammatory responses [38]. Not all immune responses to exercise are affected in the same way by cytomegalovirus, however. Exercise-induced immune surveillance by natural killer cells is impaired, whereas exercise-induced B-cell responses remain unaffected, among people latently infected with cytomegalovirus [38].

Further justification for promoting a physically active lifestyle among older adults is that habitual physical activity declines with aging, due to an increase in time spent undertaking sedentary behaviors and decreasing time spent undertaking moderate-to-vigorous physical activity [39], leading to muscle wasting and weakness [40]. Skeletal muscle is a major immunoregulatory organ and produces a range of soluble factors, which have anti-inflammatory and immunoprotective effects [41]. Indeed, regular exercise improves physical capacity and muscle mass and function and reduces cardiometabolic risk factors and systemic inflammation in older adults [42]. Physically active older adults have lower concentrations of circulating proinflammatory biomarkers, and higher concentrations of anti-inflammatory cytokines, compared to physically inactive individuals, and this is partly due to muscle physiology [42]. Indeed, muscle contraction triggers an anti-inflammatory response by releasing so-called "myokines" - muscle-secreted factors that influence almost all aspects of physiology and metabolism [43]. During exercise, predominantly IL-6 is released into

Physical Inactivity, Aging, and COVID-19 the blood from contracting skeletal muscle, independently of TNF- $\alpha$ or NF- $\kappa B$ activation (e.g., as opposed to sepsis) and induces a subsequent increase in the production of IL-1ra and IL- 10 by various immune cells. In addition to the acute effects brought about by stimulating the "myokinome," regular physical activity also modulates the properties of subcutaneous and visceral adipose tissue, reducing systemic inflammation [28]. Thus, partly due to these mechanisms, physical activity is associated with a reduced incidence of chronic age-related conditions, such as cardiovascular, metabolic and pulmonary diseases, and cancer [44]. In addition, these anti-inflammatory mechanisms provide a nonpharmacological therapeutic intervention that can influence the disease pathogenesis and disease trajectory in a number of clinical conditions $[45,46]$. Taken together, these data emphasize the importance of maintaining optimal levels of physical activity in older adults in order to ameliorate (or perhaps prevent) age-related disturbances in the immune system and minimize complications caused by chronic disease.

Given the very recent emergence of SARS-CoV-2 and COVID-19, future studies should investigate whether being physically active is a protective factor for reducing the risk of complications caused by COVID-19. This hypothesis is supported by a recent cohort study that calculated the population attributable fraction to show that physical inactivity accounted for up to $8.6 \%$ of hospitalized cases of COVID-19, and obesity - a condition potentially preventable by physical activity - contributed to $29.5 \%$ of the cases [47]. Interventional studies are necessary to confirm these findings. In addition, it should be examined whether people who are regularly physically active exhibit faster recovery times and are less likely to have poor outcomes. Considering that single bouts of aerobic and resistance exercise have been shown to improve vaccine responses [26], if older adults exhibiting signs of immunosenescence generate poor antibody responses to vaccines developed for SARS-CoV-2 [36], then exercise could be prescribed at the time of vaccine administration to boost antibody responses as a form of "behavioral adjuvant," i.e., the socalled immune-enhancement hypothesis [48], which remains to be tested in the context of COVID-19.

\section{Summary and Conclusions: the Benefits of Remaining Active during Isolation}

Social isolation is likely to lead to a decline in physical activity, which could result in immune dysregulation but more broadly also impairments in glucose and 


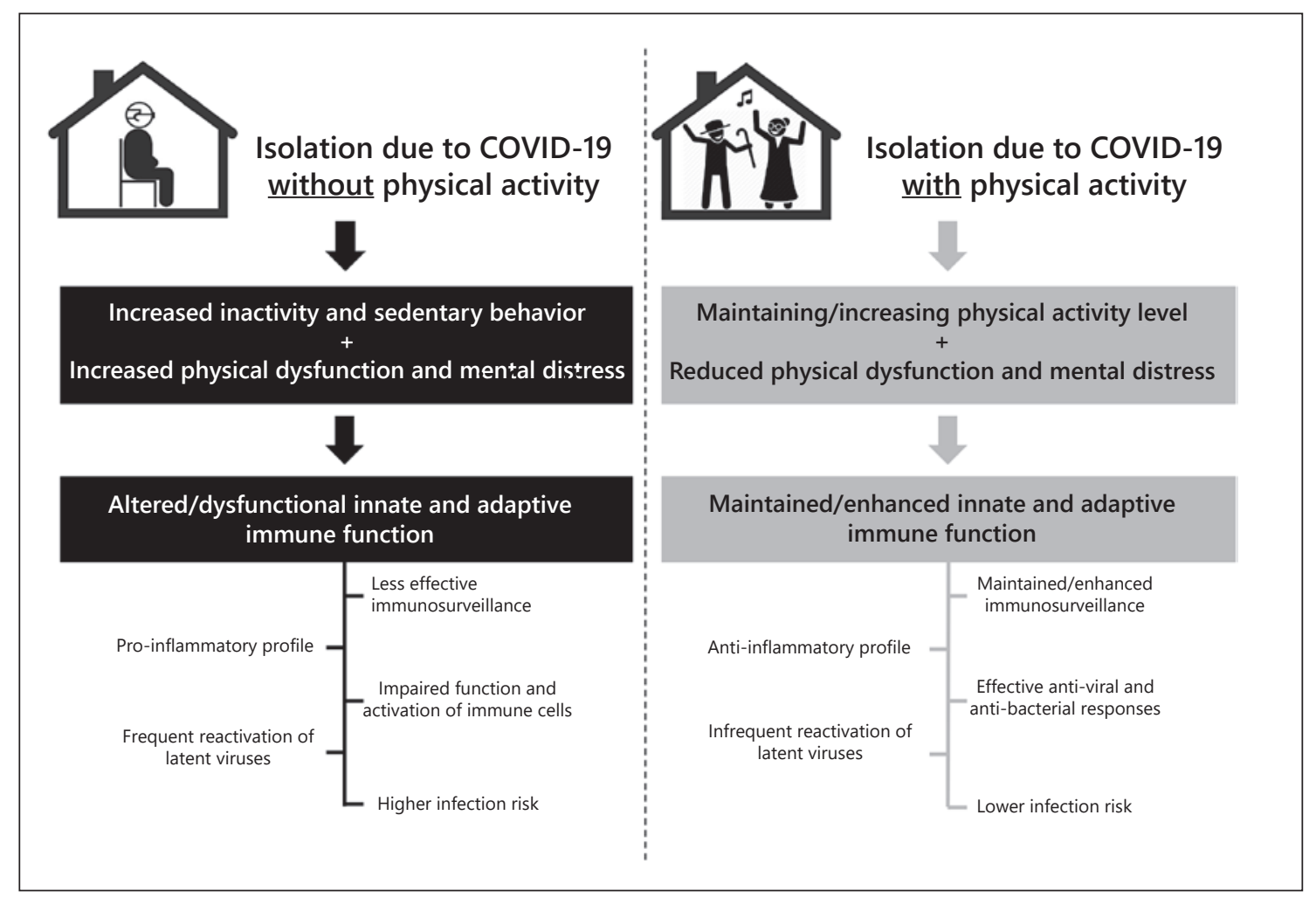

Fig. 2. Potential impact of isolation throughout the COVID-19 pandemic on immune function, with or without physical activity.

lipid metabolism, physical dysfunction, and mental distress [49]. In this scenario, the maintenance of physical activity levels become of paramount relevance for improving immune function for the general population (Fig. 2). Physical activity is particularly important for older adults and those with chronic disease not only because these individuals may exhibit impaired immune function [26] but also because these people will gain almost immediate benefits related to cardiometabolic health, muscle function, and overall wellbeing $[12,49]$. Of clinical relevance, social isolation has been associated with heart disease, depression, anxiety, and mental distress [50]. Some studies have shown that stress, depression symptoms, and social isolation can impair immune defenses, which can make an individual more susceptible to a potential viral infection $[13,27]$. In this context, promoting a physically active lifestyle could help individuals to cope with the psychological distress and the dramatic lifestyle changes during social isolation. Although it is worth emphasizing that there is no direct evidence that physical activity can prevent or treat COVID-19, promoting an active lifestyle is a key intervention to counteract the effects of social isolation.
Thus, healthcare professionals and public health policy makers should promote physical activity to everyone, but especially older adults and other at-risk individuals, such as those living with chronic diseases associated with aging and lifestyle.

\section{Conflict of Interest Statement}

The authors declare no conflict of interests.

\section{Funding Sources}

A.D., A.J.P., and B.G. are supported by grants from the Fundação de Amparo à Pesquisa do Estado de São Paulo (FAPESP; 2019/22160-6; 2015/26937-4 and 2018/19418-9; and 2017/135522).

\section{Author Contributions}

A.D., A.J.P., and B.G. drafted this paper with critical revision from J.E.T. All of the authors have seen and approved the final version of this papers for publication. 


\section{References}

1 Tay MZ, Poh CM, Rénia L, MacAry PA, Ng LF. The trinity of COVID-19: immunity, inflammation and intervention. Nat Rev Immunol. 2020 Jun;20(6):363-74.

2 Werneck AO, Collings PJ, Barboza LL, Stubbs B, Silva DR. Associations of sedentary behaviors and physical activity with social isolation in 100,839 school students: The Brazilian Scholar Health Survey. Gen Hosp Psychiatry. 2019 Jul-Aug;59:7-13.

3 Flynn MG, Markofski MM, Carrillo AE. Elevated Inflammatory Status and Increased Risk of Chronic Disease in Chronological Aging: inflamm-aging or Inflamm-inactivity? Aging Dis. 2019 Feb;10(1):147-56.

4 Duggal NA, Pollock RD, Lazarus NR, Harridge S, Lord JM. Major features of immunesenescence, including reduced thymic output, are ameliorated by high levels of physical activity in adulthood. Aging Cell. 2018 Apr; 17(2):e12750.

5 Duggal NA, Niemiro G, Harridge SD, Simpson RJ, Lord JM. Can physical activity ameliorate immunosenescence and thereby reduce age-related multi-morbidity? Nat Rev Immunol. 2019 Sep;19(9):563-72.

6 Shephard RJ. Development of the discipline of exercise immunology. Exerc Immunol Rev. 2010;16:194-222.

7 Jung YS, Park JH, Park DI, Sohn CI, Lee JM, Kim TI. Physical Inactivity and Unhealthy Metabolic Status Are Associated with Decreased Natural Killer Cell Activity. Yonsei Med J. 2018 Jun;59(4):554-62.

8 Murdaca G, Setti M, Brenci S, Fenoglio D, Lantieri P, Indiveri F, et al. Modifications of immunological and neuro-endocrine parameters induced by antiorthostatic bed-rest in human healthy volunteers. Minerva Med. 2003 Dec;94(6):363-78.

9 Uchakin PN, Stowe RP, Paddon-Jones D, Tobin BW, Ferrando AA, Wolfe RR. Cytokine secretion and latent herpes virus reactivation with 28 days of horizontal hypokinesia. Aviat Space Environ Med. 2007 Jun; 78(6):608-12.

10 Kelsen J, Bartels LE, Dige A, Hvas CL, FringsMeuthen P, Boehme G, et al. 21 Days headdown bed rest induces weakening of cell-mediated immunity - Some spaceflight findings confirmed in a ground-based analog. Cytokine. 2012 Aug;59(2):403-9.

11 Simpson RJ, Kunz H, Agha N, Graff R. Exercise and the Regulation of Immune Functions. Prog Mol Biol Transl Sci. 2015;135: 355-80.

12 Hoff P, Belavý DL, Huscher D, Lang A, Hahne M, Kuhlmey AK, et al. Effects of 60-day bed rest with and without exercise on cellular and humoral immunological parameters. Cell Mol Immunol. 2015 Jul;12(4):483-92.

13 Sonnenfeld G. The immune system in space: Including Earth-based benefits of spacebased research. Curr Pharm Biotechnol. 2005 Aug;6(4):343-9.
14 Aviles H, Belay T, Fountain K, Vance M, Sonnenfeld G. Increased susceptibility to Pseudomonas aeruginosa infection under hindlimbunloading conditions. J Appl Physiol (1985). 2003 Jul;95(1):73-80.

15 Mehta SK, Crucian B, Pierson DL, Sams C, Stowe RP. Monitoring immune system function and reactivation of latent viruses in the Artificial Gravity Pilot Study. J Gravit Physiol. 2007 Jul;14(1):21-5.

16 Clemens R, Sänger R, Kruppenbacher J, Höbel W, Stanbury W, Bock HL, et al. Booster immunization of low- and non-responders after a standard three dose hepatitis B vaccine schedule-results of a post-marketing surveillance. Vaccine. 1997 Mar;15(4):349-52.

17 Eliakim A, Schwindt C, Zaldivar F, Casali P, Cooper DM. Reduced tetanus antibody titers in overweight children. Autoimmunity. 2006 Mar;39(2):137-41.

18 Sheridan PA, Paich HA, Handy J, Karlsson EA, Hudgens MG, Sammon AB, et al. Obesity is associated with impaired immune response to influenza vaccination in humans. Int J Obes. 2012 Aug;36(8):1072-7.

19 Nieman DC, Henson DA, Nehlsen-Cannarella SL, Ekkens M, Utter AC, Butterworth DE, et al. Influence of obesity on immune function. J Am Diet Assoc. 1999 Mar;99(3):294-9.

20 Gottschlich MM, Mayes T, Khoury JC, Warden GD. Significance of obesity on nutritional, immunologic, hormonal, and clinical outcome parameters in burns. J Am Diet Assoc. 1993 Nov;93(11):1261-8.

21 Falagas ME, Kompoti M. Obesity and infection. Lancet Infect Dis. 2006 Jul;6(7):438-46.

22 Campbell JP, Turner JE. Debunking the myth of exercise-induced immune suppression: redefining the impact of exercise on immunological health across the lifespan. Front Immunol. 2018 Apr;9:648.

23 Nieman DC, Wentz LM. The compelling link between physical activity and the body's defense system. J Sport Health Sci. 2019 May; 8(3):201-17.

24 Simpson RJ, Campbell JP, Gleeson M, Krüger K, Nieman DC, Pyne DB, et al. Can exercise affect immune function to increase susceptibility to infection? Exerc Immunol Rev. 2020; 26:8-22.

25 von der Weid PY. Lymphatic vessel pumping. Adv Exp Med Biol. 2019;1124:357-77.

26 Turner JE. Is immunosenescence influenced by our lifetime "dose" of exercise? Biogerontology. 2016 Jun;17(3):581-602.

27 Crucian BE, Choukèr A, Simpson RJ, Mehta S, Marshall G, Smith SM, et al. Immune system dysregulation during spaceflight: potential countermeasures for deep space exploration missions. Front Immunol. 2018 Jun;9: 1437.

28 Gleeson M, Bishop NC, Stensel DJ, Lindley MR, Mastana SS, Nimmo MA. The anti-inflammatory effects of exercise: mechanisms and implications for the prevention and treat- ment of disease. Nat Rev Immunol. 2011 Aug; 11(9):607-15.

29 Krüger K, Lechtermann A, Fobker M, Völker K, Mooren FC. Exercise-induced redistribution of T lymphocytes is regulated by adrenergic mechanisms. Brain Behav Immun. 2008 Mar;22(3):324-38.

30 Nikolich-Žugich J. The twilight of immunity: emerging concepts in aging of the immune system. Nat Immunol. 2018 Jan;19(1):10-9.

31 Crooke SN, Ovsyannikova IG, Poland GA, Kennedy RB. Immunosenescence and human vaccine immune responses. Immun Ageing. 2019 Sep;16(1):25.

32 Johnson RW, Bouhassira D, Kassianos G, Leplège A, Schmader KE, Weinke T. The impact of herpes zoster and post-herpetic neuralgia on quality-of-life. BMC Med. 2010 Jun; 8(1):37.

33 Gordon A, Reingold A. The Burden of Influenza: a Complex Problem. Curr Epidemiol Rep. 2018;5(1):1-9.

34 Wagner A, Garner-Spitzer E, Jasinska J, Kollaritsch H, Stiasny K, Kundi M, et al. Age-related differences in humoral and cellular immune responses after primary immunisation: indications for stratified vaccination schedules. Sci Rep. 2018 Jun;8(1):9825.

35 Pawelec G. Age and immunity: what is "immunosenescence”? Exp Gerontol. 2018 May; 105:4-9.

36 Pawelec G, Weng NP. Can an effective SARSCoV-2 vaccine be developed for the older population? Immun Ageing. 2020 Apr;17(1): 8.

37 Wong GC, Narang V, Lu Y, Camous X, Nyunt MS, Carre C, et al. Hallmarks of improved immunological responses in the vaccination of more physically active elderly females. Exerc Immunol Rev. 2019;25:20-33.

38 Simpson RJ, Bigley AB, Spielmann G, LaVoy EC, Kunz H, Bollard CM. Human cytomegalovirus infection and the immune response to exercise. Exerc Immunol Rev. 2016;22:8-27.

39 Hallal PC, Andersen LB, Bull FC, Guthold R, Haskell W, Ekelund U; Lancet Physical Activity Series Working Group. Global physical activity levels: surveillance progress, pitfalls, and prospects. Lancet. 2012 Jul;380(9838): 247-57.

40 Cunningham C, O’ Sullivan R, Caserotti P, Tully MA. Consequences of physical inactivity in older adults: A systematic review of reviews and meta-analyses. Scand J Med Sci Sports. 2020 May;30(5):816-27.

41 Pedersen BK, Febbraio MA. Muscles, exercise and obesity: skeletal muscle as a secretory organ. Nat Rev Endocrinol. 2012 Apr;8(8):45765.

42 Ferrer MD, Capó X, Martorell M, BusquetsCortés C, Bouzas C, Carreres S, et al. Regular Practice of Moderate Physical Activity by Older Adults Ameliorates Their Anti-Inflammatory Status. Nutrients. 2018 Nov; 10(11):E1780. 
43 Benatti FB, Pedersen BK. Exercise as an antiinflammatory therapy for rheumatic diseasesmyokine regulation. Nat Rev Rheumatol. 2015 Feb;11(2):86-97.

44 Booth FW, Roberts CK, Laye MJ. Lack of exercise is a major cause of chronic diseases. Compr Physiol. 2012 Apr;2(2):1143-211.

45 Whitham M, Febbraio MA. The ever-expanding myokinome: discovery challenges and therapeutic implications. Nat Rev Drug Discov. 2016 Oct;15(10):719-29.
46 Pinto AJ, Roschel H, de Sá Pinto AL, Lima FR, Pereira RM, Silva CA, et al. Physical inactivity and sedentary behavior: overlooked risk factors in autoimmune rheumatic diseases? Autoimmun Rev. 2017 Jul;16(7):667-74.

47 Hamer M, Kivimäki M, Gale CR, David Batty G. Lifestyle risk factors, inflammatory mechanisms, and COVID-19 hospitalization: A community-based cohort study of 387,109 adults in UK. Brain Behav Immun. 2020 May;S0889-1591(20)30996-X.
48 Edwards KM, Burns VE, Carroll D, Drayson $\mathrm{M}$, Ring $\mathrm{C}$. The acute stress-induced immunoenhancement hypothesis. Exerc Sport Sci Rev. 2007 Jul;35(3):150-5.

49 Lauder W, Mummery K, Jones M, Caperchione $\mathrm{C}$. A comparison of health behaviours in lonely and non-lonely populations. Psychol Health Med. 2006 May;11(2):233-45.

50 Schrempft S, Jackowska M, Hamer M, Steptoe A. Associations between social isolation, loneliness, and objective physical activity in older men and women. BMC Public Health. 2019 Jan;19(1):74. 